\title{
The m-Point Quaternary Approximating Subdivision Schemes
}

\author{
Shahid S. Siddiqi ${ }^{1}$, Muhammad Younis ${ }^{1,2}$ \\ ${ }^{1}$ Department of Mathematics, University of the Punjab, Lahore, Pakistan \\ ${ }^{2}$ Centre for Undergraduate Studies, University of the Punjab, Lahore, Pakistan \\ Email: shahidsiddiqiprof@yahoo.co.uk, younis.pu@gmail.com
}

Received January 18, 2013; revised February 19, 2013; accepted March 11, 2013

Copyright (C) 2013 Shahid S. Siddiqi, Muhammad Younis. This is an open access article distributed under the Creative Commons Attribution License, which permits unrestricted use, distribution, and reproduction in any medium, provided the original work is properly cited.

\begin{abstract}
In this article, the objective is to introduce an algorithm to produce the quaternary $m$-point (for any integer $m>1$ ) approximating subdivision schemes, which have smaller support and higher smoothness, comparing to binary and ternary schemes. The proposed algorithm has been derived from uniform B-spline basis function using the Cox-de Boor recursion formula. In order to determine the convergence and smoothness of the proposed schemes, the Laurent polynomial method has been used.
\end{abstract}

Keywords: Cox-De Boor Recursion Formula; Quaternary; Approximating Subdivision Schemes; Convergence and Smoothness

\section{Introduction}

Until a few years ago all the work in the area of univariate subdivision was limited to consider just binary (Chaikin [1]; Dyn et al. [2,3]; Siddiqi and Younis [4]; Beccari et al. [5]) and ternary (Hassan and Dodgson [6]; Hassan et al. [7]; Ko et al. [8]; Mustafa et al. [9]) scenarios. In recent time, some proposals of quaternary subdivision schemes have introduced new interest in the era of subdivision, showing the possibility of treating refinement schemes with arity other than two or three.

Since subdivision schemes propose efficient iterative algorithms to produce the smooth curves and surfaces from a discrete set of control points by subdividing them according to some refining rules, recursively. These refining rules are very helpful and useful for the creation of smooth curves and surfaces in computational geometry and geometric designing due to their wide range of applications in many areas like engineering, medical science and image processing etc.

In this article an algorithm has been introduced to produce the quaternary $m$-point (for any integer $m>1$ ) approximating subdivision schemes. This algorithm has been developed using the Cox-de Boor recursion formula, in the form of uniform B-spline blending functions to produce piecewise polynomials of order $p$ over the interval $t \in[0,1]$ (for detail, see Section 2).
The quaternary subdivision scheme can be defined in terms of a mask consisting of a finite set of non-zero coefficients $\boldsymbol{a}=\left\{a_{i}: i \in Z\right\}$ as follows

$$
f_{i}^{k+1}=\sum_{j \in Z} a_{i-4 j} f_{j}^{k}, i \in Z .
$$

The formal definitions and the notion for the convergence analysis of the quaternary subdivision scheme are as follows:

The quaternary convergent subdivision scheme $S$ with the corresponding mask $\left\{a_{i}: i \in Z\right\}$ necessarily satisfies

$$
\sum_{j \in Z} a_{4 j}=\sum_{j \in Z} a_{4 j+1}=\sum_{j \in Z} a_{4 j+2}=\sum_{j \in Z} a_{4 j+3}=1 .
$$

It follows that the symbol of a convergent subdivision scheme satisfies the conditions $a\left(\mathrm{e}^{\frac{n i \pi}{4}}\right)=0$ and $a(1)=4$, for $n=1,2,3$.

Introducing a symbol called the Laurent polynomial

$$
a(z)=\sum_{i \in Z} a_{i} z^{i},
$$

of a mask $\left\{a_{i}: i \in Z\right\}$ with finite support. In view of Dyn [3], the sufficient and necessary conditions for a uniform convergent scheme are defined as follows.

A subdivision scheme $S$ is uniform convergent if 
and only if there is an integer $L \geq 1$, such that

$$
\left\|\left(\frac{1}{4} S_{1}\right)^{L}\right\|_{\infty}<1
$$

subdivision $S_{1}$ with symbol $a_{1}(z)$ is related to $S$ with symbol $a(z)$, where $a_{1}(z)=\frac{4 z^{3}}{1+z+z^{2}+z^{3}} a(z)$ and satisfying the property

$$
\mathrm{d} f^{k}=S_{1} \mathrm{~d} f^{k-1}, k=1,2, \cdots,
$$

where $f^{k}=S^{k} f^{0}=\left\{f_{i}^{k}: i \in Z\right\}$ and

$\mathrm{d} f^{k}=\left\{\left(\mathrm{d} f^{k}\right)_{i}=4^{k}\left(f_{i+1}^{k}-f_{i}^{k}\right): i \in Z\right\}$. The norm $\|S\|_{\infty}$ of a subdivision scheme $S$ with a mask $\left\{a_{i}, i \in Z\right\}$ is defined by

$$
\|S\|_{\infty}=\max \left\{\sum_{i}\left|a_{4 i}\right|, \sum_{i}\left|a_{4 i+1}\right|, \sum_{i}\left|a_{4 i+2}\right|, \sum_{i}\left|a_{4 i+3}\right|\right\}
$$

and

$$
\left\|\left(\frac{1}{4} S\right)^{L}\right\|_{\infty}=\max \left\{\sum_{\beta}\left|b_{\gamma+4^{L} \beta}^{[L]}\right|: \gamma=0,1, \cdots, 4^{L}-1\right\},
$$

where

$$
b^{[m, L]}(z)=\frac{1}{4^{L}} a_{m}^{[L]}(z), m=1,2, \cdots, L,
$$

where

$a_{m}(z)=\left(\frac{4 z^{3}}{1+z+z^{2}+z^{3}}\right) a_{m-1}(z)=\left(\frac{4 z^{3}}{1+z+z^{2}+z^{3}}\right)^{m} a(z)$

and

$$
a_{m}^{[L]}(z)=\prod_{j=0}^{L-1} a_{m}\left(z^{4^{j}}\right) .
$$

The paper is organized as follows, in Section 2 the algorithm to construct $m$-point (for any integer $m>1$ ) quaternary schemes has been introduced. Three examples are considered to produce the masks of 2-point (corner cutting), 3-point and 4-point schemes in the same section. In Section 3, the polynomial reproduction property has been discussed. The conclusion is drawn in Section 4.

\section{Construction of the Algorithm}

In this section, an algorithm has been constructed to produce the quaternary $m$-point approximating subdivision schemes using the uniform B-spline basis functions and the Cox-de Boor recursion relation. The Cox-de Boor recursion relation, in view of Buss [10], can be defined as follows:
The recursion relation is the generalization to B-spline of degree $k$ (or of order $n$, i.e., $k=n-1$ ). For this, consider $T$ to be a set of $l+1$ non-decreasing real numbers in such a way that $t_{0} \leq t_{1} \leq t_{2} \leq \cdots \leq t_{l}$. The values $t_{i}$ 's, not necessarily uniformly spaced, are called knots of non-uniform spline and the set $T$ is called knot vector. The uniform B-splines are just the special case of non-uniform B-splines in which the knots are equally spaced such that $t_{i+1}-t_{i}$ is a constant for $0 \leq i \leq n-1$ (i.e., $\left.t_{i}=i\right)$. Note that the blending functions $N_{i, n}(t)$ of or- der $n$ depend only on the knot positions and are de- fined by induction on $n \geq 1$ as follows.

First, for $i=0,1, \cdots, l-1$, let

$$
N_{i, 1}(t)=\left\{\begin{array}{l}
1, t \in\left[t_{i}, t_{i+1}\right), \\
0, \text { otherwise. }
\end{array}\right.
$$

Second for $n \geq 1$. Setting $n=k+1, N_{i, k+1}(t)$ is defined by the Cox-de Boor formula as,

$$
N_{0, k+1}(t)=\frac{t-t_{i}}{t_{i+k}-t_{i}} N_{i, k}(t)+\frac{t_{i+k+1}-t}{t_{i+k+1}-t_{i+1}} N_{i+1, k}(t) .
$$

The form of above recursive formulas for the blending function immediately implies that the functions $N_{i, n}(t)$ are piecewise polynomials of degree $n-1$ and that the breaks between pieces occur at the knots $t_{i}$.

In view of above recursion formula, the Uniform Bspline blending functions $N_{0, p}(t)$ of order $p$ over the interval $t \in[0,1]$, together with the properties [10], can be defined in Equation (2).

The blending functions must satisfy the following properties:

- The blending functions are translates of each other, that is, $N_{i}(t)=N_{0}(t-i)$.

- The blending functions are a partition of unity, that is, $\sum_{i} N_{i}(t)=1$.

- $0 \leq N_{i}(t) \leq 1$ for all $t$.

- The functions $N_{i}(t)$ have continuous $p-1$ derivatives, that is, they are $C^{p-1}$-continuous.

$$
N_{0, p}(t)=\frac{t}{p-1} N_{0, p-1}(t)+\frac{p-t}{p-1} N_{0, p-1}(t-1) .
$$

The masks $a_{i}, i=0,1,2, \cdots, m-1$ of the proposed quaternary $m$-point scheme can be calculated using the following recurrence relation

$$
\begin{aligned}
& a_{i}=N_{0, m}\left((m-i)-\frac{7}{8}\right), \text { and } b_{i}=N_{0, m}\left((m-i)-\frac{5}{8}\right), \\
& i=0,1,2, \cdots, m-1,
\end{aligned}
$$

where $N_{0, m}(t)$ is a uniform B-spline basis function of degree $m-1$. In the following, some examples are considered to produce the masks of 2-point, 3-point and 4-point quaternary approximating schemes after setting 
$m=2,3$ and 4, respectively, in recurrence relation (3).

The 2-point scheme: To obtain the mask of quarternary 2-point scheme, set $m=2$ in above relation (3). It may be noted that the linear uniform B-spline basis function $N_{0,2}(t)$ produces the mask of 2-point quarternary scheme (which is also called corner cutting scheme). Thus 2-point scheme (after adjusting the mask) to refine the control polygon is defined as follows:

$$
\left.\begin{array}{rl}
f_{4 i}^{k+1} & =\frac{1}{8} f_{i}^{k}+\frac{7}{8} f_{i+1}^{k} \\
f_{4 i+1}^{k+1} & =\frac{3}{8} f_{i}^{k}+\frac{5}{8} f_{i+1}^{k} \\
f_{4 i+2}^{k+1} & =\frac{5}{8} f_{i}^{k}+\frac{3}{8} f_{i+1}^{k} \\
f_{4 i+3}^{k+1} & =\frac{7}{8} f_{i}^{k}+\frac{1}{8} f_{i+1}^{k}
\end{array}\right\}
$$

Now, the convergence and smoothness of the proposed 2-point scheme can be analyzed using the Laurent polynomial method introduced by Tang et al. [11].

Theorem 2.1: The quaternary 2-point approximating subdivision scheme converges and has smoothness $C^{1}$.

Proof. To prove that the subdivision scheme $S$ corresponding to the symbol $a(z)$ is $C^{1}$. So, the Laurent polynomial $a(z)$ for the mask of the scheme can be written as

$$
\begin{aligned}
a(z)= & \frac{1}{8} z^{-4}+\frac{3}{8} z^{-3}+\frac{5}{8} z^{-2}+\frac{7}{8} z^{-1} \\
& +\frac{7}{8}+\frac{5}{8} z^{1}-\frac{3}{8} z^{2}-\frac{1}{8} z^{3}
\end{aligned}
$$

The Laurent polynomial method is used to prove the smoothness of the scheme to be $C^{1}$. Taking

$$
b^{[m, L]}(z)=\frac{1}{4^{L}} a_{m}^{[L]}(z), m=1,2, \cdots, L,
$$

where

$$
\begin{aligned}
a_{m}(z) & =\left(\frac{4 z^{2}}{1+z+z^{2}+z^{3}}\right) a_{m-1}(z) \\
& =\left(\frac{4 z^{2}}{1+z+z^{2}+z^{3}}\right)^{m} a(z)
\end{aligned}
$$

and

$$
a_{m}^{[L]}(z)=\prod_{j=0}^{L-1} a_{m}\left(z^{4^{j}}\right) .
$$

With a choice of $m=1$ and $L=1$, it can be written as

$$
\begin{aligned}
b^{[1,1]}(z) & =\frac{1}{4^{1}} a_{1}(z) \\
& =1+\frac{1}{2} z^{-1}+z^{1}+z^{2}+\frac{1}{2} z^{3} .
\end{aligned}
$$

Since the norm of subdivision $\frac{1}{4} S_{1}$ is

$$
\begin{aligned}
& \left\|\frac{1}{4} S_{1}\right\|_{\infty} \\
& =\max \left\{\sum_{\beta}\left|b_{\gamma+4 \beta}^{[1,1]}\right|: \gamma=0,1,2,3\right\} \\
& =\max \left\{\frac{1}{4}, \frac{1}{4}, \frac{1}{4}, \frac{1}{4}\right\}=\frac{1}{4}<1,
\end{aligned}
$$

therefore $\frac{1}{4} S_{1}$ is contractive, by Theorem 3 , and so $S$ is convergent.

In order to prove the scheme developed to be $C^{1}$, consider $m=2$ and $L=1$; it can be written as

$$
b^{[2,1]}(z)=\frac{1}{4^{1}} a_{2}(z)=2 z^{2}+2 z^{3} .
$$

Since the norm of subdivision $\frac{1}{4} S_{2}$ is

$$
\begin{aligned}
\left\|\frac{1}{4} S_{2}\right\|_{\infty} & =\max \left\{\sum_{\beta}\left|b_{\gamma+4 \beta}^{[2,1]}\right|: \gamma=0,1,2,3\right\} \\
& =\max \left\{0,0, \frac{1}{2}, \frac{1}{2}\right\}=\frac{1}{2}<1,
\end{aligned}
$$

therefore $\frac{1}{4} S_{2}$ is contractive. Consequently, $S_{1}$ is convergent and $S \in C^{1}$.

The 3-point scheme: To obtain the mask of quarternary univariate 3 -point scheme, set $m=3$ in recursion relation (3). The quadratic uniform $\mathrm{B}$-spline basis functions $N_{0,3}(t)$ are obtained. The mask $a_{i}, i=0,1,2$ of the proposed quaternary 3 -point scheme can be calculated from these basis functions. The 3-point scheme (after adjusting the mask), to refine the control polygon, is defined as:

$$
\left.\begin{array}{rl}
f_{4 i}^{k+1} & =\frac{49}{128} f_{i-1}^{k}+\frac{39}{64} f_{i}^{k}+\frac{1}{128} f_{i+1}^{k} \\
f_{4 i+1}^{k+1} & =\frac{25}{128} f_{i-1}^{k}+\frac{47}{64} f_{i}^{k}+\frac{9}{128} f_{i+1}^{k} \\
f_{4 i+2}^{k+1} & =\frac{9}{128} f_{i-1}^{k}+\frac{47}{64} f_{i}^{k}+\frac{25}{128} f_{i+1}^{k} \\
f_{4 i+3}^{k+1} & =\frac{1}{128} f_{i-1}^{k}+\frac{39}{64} f_{i}^{k}+\frac{49}{128} f_{i+1}^{k}
\end{array}\right\}
$$

Theorem 2.2: The quaternary 3-point approximating subdivision scheme converges and has smoothness $C^{2}$.

Proof. The smoothness of the above subdivision scheme can be calculated following the same procedure.

The 4-point scheme: Now, a 4-point quaternary scheme is presented and masks of the scheme can be cal- 
culated from the cubic basis function. After setting $m=4$ in relation (3) the cubic B-spline basis functions $N_{0,4}(t)$ can be calculated. Thus, 4-point scheme is defined as follows

$$
\left.\begin{array}{rl}
f_{4 i}^{k+1} & =\frac{341}{3072} f_{i-1}^{k}+\frac{2003}{3072} f_{i}^{k}+\frac{725}{3072} f_{i+1}^{k}+\frac{1}{3072} f_{i+2}^{k} \\
f_{4 i+1}^{k+1} & =\frac{125}{3072} f_{i-1}^{k}+\frac{1697}{3072} f_{i}^{k}+\frac{1223}{3072} f_{i+1}^{k}+\frac{27}{3072} f_{i+2}^{k} \\
f_{4 i+2}^{k+1} & =\frac{27}{3072} f_{i-1}^{k}+\frac{1223}{3072} f_{i}^{k}+\frac{1697}{3072} f_{i+1}^{k}+\frac{125}{3072} f_{i+2}^{k} \\
f_{4 i+3}^{k+1} & =\frac{1}{3072} f_{i-1}^{k}+\frac{725}{3072} f_{i}^{k}+\frac{2003}{3072} f_{i+1}^{k}+\frac{341}{3072} f_{i+2}^{k}
\end{array}\right\}
$$

Theorem 2.3: The quaternary 4-point approximating subdivision scheme converges and has smoothness $C^{3}$.

Proof. The smoothness of the above subdivision scheme can be calculated following the same procedure.

In the following section the polynomial reproduction property has been discussed.

\section{Properties}

The polynomial reproduction property has its own importance. As, the reproduction property of the polynomials up to certain degree $d$ implies that the scheme has $d+1$ approximation order. For this, polynomial reproduction can be made from initial date which has been sampled from some polynomial function. In view of [12], the polynomial reproduction property of the proposed scheme can be obtained after having the parametrization $\tau$ and definitions in the following manner.

Definition 3.1: For quaternary subdivision scheme the parametrization $\tau=a^{\prime}(1) / 4$ the corresponding parametric shift and attach the data $f_{i}^{k}$ for $i \in Z, k \in N$ to the parameter values

$$
t_{i}^{k}=t_{0}^{k}+\frac{i}{4^{k}} \text { with } t_{0}^{k}=t_{0}^{k-1}-\frac{\tau}{4^{k}} .
$$

Definition 3.2: A quaternary subdivision scheme reproduces polynomial of degree $d$ if it is convergent and its continuous limit function (for any polynomial $\left.p \in \pi_{d}\right)$ is equal to $p$ and initial data $f_{i}^{0}=p\left(t_{i}^{0}\right), i \in Z$.

Theorem 3.3: A convergent quaternary subdivision scheme reproduces polynomials of degree $d$ with respect to the parametrization defined in (8) if and only if

$$
\begin{aligned}
& a^{(k)}(1)=4 \prod_{l=0}^{k-1}(\tau-l) \text { and } a^{(k)}\left(\mathrm{e}^{\frac{2 n i \pi}{4}}\right)=0 \\
& \text { for } k=0,1, \cdots, d \text { and } n=1,2,3 \text {. }
\end{aligned}
$$

Proof. The induction over $d$ can be performed to prove this theorem following [12].

In view of [12], the following proposition helps to find the necessary conditions defined in (9).

Proposition 3.4: Let $d \in N$ and $\tau \in R$. Then a subdivision symbol $a(z)$ satisfies

$$
a^{(k)}(1)=4 \prod_{l=0}^{k-1}(\tau-l) \text { for } k=0,1, \cdots, d
$$

if and only if $b(z)=a\left(z^{4}\right) z^{-4 \tau}$ satisfies

$$
b(1)=4 \text { and } b^{(k)}(1)=0 \text { for } k=0,1, \cdots, d
$$

( $k^{\text {th }}$ derivative of the symbol) which in turn is equivalent to require that $b(z)=(1-z)^{d+1} c(z)+4$ for some $c(z)$.

Proposition 3.5: Let a quaternary subdivision scheme that reproduces polynomial up to degree $d$. Then the smoothed scheme $S_{b}$ with the symbol

$$
\begin{gathered}
b(z)=\frac{1+z+z^{2}+z^{3}}{4} a(z) \text { satisfies the conditions } \\
b(1)=4 \text { and } b^{(k)}\left(\mathrm{e}^{\frac{2 n i \pi}{4}}\right)=0, k=0,1, \cdots, d+1
\end{gathered}
$$

and hence generates polynomial of degree $d+1$, but it has only linear reproduction.

Proof. Following [12], for some Laurent polynomial $b(z)$ with $b(1)=\frac{1}{4^{d}}$, we have

$$
a(z)=\left(1+z+z^{2}+z^{3}\right)^{d+1} b(z)=\left(\frac{1-z^{4}}{1-z}\right)^{d+1} b(z)
$$

and the fact $b(1)=a(1)$. Thus, the $1^{\text {st }}$ derivative of $b(z)$ is

$$
b^{\prime}(z)=\frac{1+z+z^{2}+z^{3}}{4} a^{\prime}(z)+\frac{1+2 z+3 z^{2}}{4} a(z)
$$

and correct parametric shift for $S_{b}$ is

$$
\tau_{b}=\frac{b^{\prime}(1)}{4}=\frac{1}{4} a^{\prime}(1)+\frac{3}{8} a(1)=\tau_{a}+\frac{3}{2}
$$

The $2^{\text {nd }}$ derivative of $b(z)$ is

$$
\begin{aligned}
b^{\prime \prime}(z) & =\frac{1+z+z^{2}+z^{3}}{4} a^{\prime \prime}(z)+\frac{1+2 z+3 z^{2}}{2} a^{\prime}(z) \\
& +\frac{2+6 z+12 z^{2}}{4} a(z)
\end{aligned}
$$

which produces

$$
b^{\prime \prime}(1)=a^{\prime \prime}(1)+3 a^{\prime}(z)+2 a(1)=4 \tau_{a}\left(\tau_{a}-1\right)+12 \tau_{a}+8
$$

after simplification, it can be yielded that

$$
b^{\prime \prime}(1)-4 \tau_{b}\left(\tau_{b}-1\right)=5 \neq 0 \text {. }
$$

Hence, it does not reproduce polynomials of degree $d>1$. 


\section{Conclusion}

A quaternary univariate $m$-point (for any integer $m>1$ ) approximating subdivision scheme has been developed which generates the smooth limiting curves. The construction of the quaternary scheme is associated with an algorithm of uniform B-spline basis functions developed from the Cox-de Boor recursion formula. The objective is to introduce the quaternary subdivision schemes, which have smaller support and higher smoothness, comparing to binary and ternary schemes. Moreover the polynomial reproduction property has also been discussed.

\section{REFERENCES}

[1] G. M. Chaikin, "An Algorithm for High Speed Curve Generation," Graph cuts in Computer Vision, Vol. 3, No. 4, 1974, pp. 346-349.

[2] N. Dyn, J. A. Gregory and D. Levin, "A 4-Points Interpolatory Subdivision Scheme for Curve Design," Computer Aided Geometric Design, Vol. 4, No. 4, 1987, pp. 257-268. doi:10.1016/0167-8396(87)90001-X

[3] N. Dyn, "Tutorials on Multresolution in Geometric Modelling," In: A. Iske, E. Quak and M. S. Floater, Eds., Summer School Lectures Notes Series: Mathematics and Visualization, Springer, 1995, ISBN: 3-540-43639-1.

[4] S. S. Siddiqi and M. Younis, "Construction of $m$-Point Approximating Subdivision Schemes," Applied Mathematics Letters, Vol. 26, No. 3, 2013, pp. 337-343. doi:10.1016/j.aml.2012.09.016

[5] C. Beccari, G. Casciola and L. Romani, "A Non-Stationary Uniform Tension Controlled Interpolating 4-Point scheme Reproducing Conics," Computer Aided Geomet- ric Design, Vol. 24, No. 1, 2007, pp. 1-9. doi:10.1016/j.cagd.2006.10.003

[6] M. F. Hassan and N. A. Dodgson, "Ternary and Three Point Univariate Subdivision Schemes," In: A. Cohen, J.L. Merrien and L. L. Schumaker, Eds., Curve and Surface Fitting: Sant-Malo, Nashboro Press, Brentwood, 2003, pp. 199-208.

[7] M. F. Hassan, I. P. Ivrissimtzis, N. A. Dodgson and M. A. Sabin, "An Interpolating 4-Point Ternary Stationary Subdivision Scheme," Computer Aided Geometric Design, Vol. 19, No. 1, 2002, pp. 1-18. doi:10.1016/S0167-8396(01)00084-X

[8] K. P. Ko, B.-G. Lee and G. J. Yoon, "A Ternary 4-Point Approximating Subdivision Scheme," Applied Mathematics and Computation, Vol. 190, No. 2, 2007, pp. 15631573. doi:10.1016/j.amc.2007.02.032

[9] G. Mustafa, A. Ghaffar and F. Khan, "The Odd-Point Ternary Approximating Schemes," American Journal of Computational Mathematics, Vol. 1, No. 2, 2011, pp. 111118.

[10] S. R. Buss, "3-D Computer Graphics A Mathematical Introduction with OpenGL," 1st Edition, Cambridge University Press, New York, 2003. doi:10.1017/CBO9780511804991

[11] Y. Tang, K. P. Ko and B. G. Lee, "A New Proof of the Smoothness of 4-Point Deslauriers-Dubuc Scheme," Journal of Applied Mathematics and Computing Vol. 18, No. 1-2, 2005, pp. 553-562.

[12] C. Conti and K. Hormann, "Polynomial Reproduction for Univariate Subdivision Schemes of any Arity," Journal of Approximation Theory, Vol. 163, No. 4, 2011, pp. 413437. doi:10.1016/j.jat.2010.11.002 